\title{
The Electromagnetic Field Propagation in a Spherical Core
}

\author{
Osama M. Abo-Seid ${ }^{1}$, Ghada M. Sami ${ }^{2}$ \\ ${ }^{1}$ Mathematic Department, Faculty of Science, Kafr El-Sheikh University, Kafr El-Sheikh, Egypt; ${ }^{2}$ Mathematic Department, Faculty \\ of Science, Ain shams University, Cairo, Egypt. \\ Email: aboseida@yahoo.com,g_sami2003@yahoo.com
}

Received September $15^{\text {th }}, 2012$; revised October $16^{\text {th }}, 2012$; accepted October $26^{\text {th }}, 2012$

\begin{abstract}
A simple and explicit derivation for the electric and magnetic fields in the ferromagnetic cores has been studied. An improved model for analyzing the distribution of electric and magnetic fields in a toroidal core is given. This leads to a basis system for the theoretical analysis of iron loss in the magnetic cores, so, the theoretical results have been evaluated. A method is used to perform numeric calculations of the magnetic field produced by the eddy current and displacement current due to the electric field which shield the magnetic flux from the inner portion of the core cross section. The results obtained from this work could be used to evaluate the skin effect in the conductors of a winding.
\end{abstract}

Keywords: Electromagnetic Field; Wave Propagation; Magnetic Flux; Magnetic Cores

\section{Introduction}

Considerable interest has been given to the study of electric and magnetic fields distribution in a toroidal core. Some of these studies calculate the core loss theoretically [1]. In their calculation, the magnetic field strength produced by the winding current is assumed the constant around the perimeter of the cross section. Other studies have been applied to measure the magnetic core loss [2]. Also, Abo-Seida et al. [3] gave an explicit derivation for the electromagnetic transient, Abo-Seida et al. [4] derived the transient fields of a vertical electric dipole on an M-layered dielectric medium. The transient field of the magnetic dipole on a two-layered conducting earth has also been studied [5,6]. Abo-Seida [7] has studied the far field of a vertical magnetic dipole. Wait [8] derived the electromagnetic fields of a traveling current line source.

We noticed that the diameter of the core cross section is very small compared with the innermost radius of the core. In this paper, we calculated the distributions of the electric and magnetic fields in the torodial core with spherical cross section based on Maxwell's equation as in Figure 1(a) and take the boundary conditions into consideration. The magnetomotive forces are affected by the external current, the eddy and displacement current in the area $r<R$ as in Figure 1(b), but on the boundary $r=$ $R$, the magnetic field intensity is determined only by the exciting current. The induced eddy current and displacement current due to the electric field will shield the flux from the inner portion of the core section resulting in a flux skin effect analogous to the skin effect in the conductors of winding at high frequency. With the increase of frequency, this phenomenon becomes more obvious.

\section{Formulation of the Problem}

The physical model is illustrated in Figures 1(a) and (b). A frequency domain analysis was performed in this work. It is assumed that all field vectors and all currents and charge densities vary sinusoidally with time at a single angular frequency. Maxwell's equations are then written as follows:

$$
\begin{gathered}
\nabla \wedge H=J+j \omega D \\
\nabla \wedge E=-j \omega B \\
\nabla \cdot B=0 \\
\nabla \cdot D=\rho
\end{gathered}
$$

Taking the curl of (1) and substituting (2), we obtain

$$
\nabla \wedge \nabla \wedge H=\left(-j \mu \sigma \omega+\mu \varepsilon \omega^{2}\right) H
$$

where

$$
\nabla \wedge \nabla \wedge H=K^{2} H \text { and } k=\sqrt{\left(-j \mu \sigma \omega+\mu \varepsilon \omega^{2}\right)}
$$

Equation (5) can be written in spherical coordinates $(r, \theta, \varphi)$ as

$$
\begin{aligned}
& \frac{1}{r^{2}} \frac{\partial}{\partial r} r^{2} \frac{\mathrm{d} H}{\mathrm{~d} r}+\frac{1}{r^{2}}\left[\frac{1}{\sin \theta} \frac{\partial}{\partial \theta} \sin \theta \frac{\partial}{\partial \theta}+\frac{1}{\sin ^{2} \theta} \frac{\partial^{2}}{\partial \varphi^{2}}\right] H \\
& +k H=0
\end{aligned}
$$




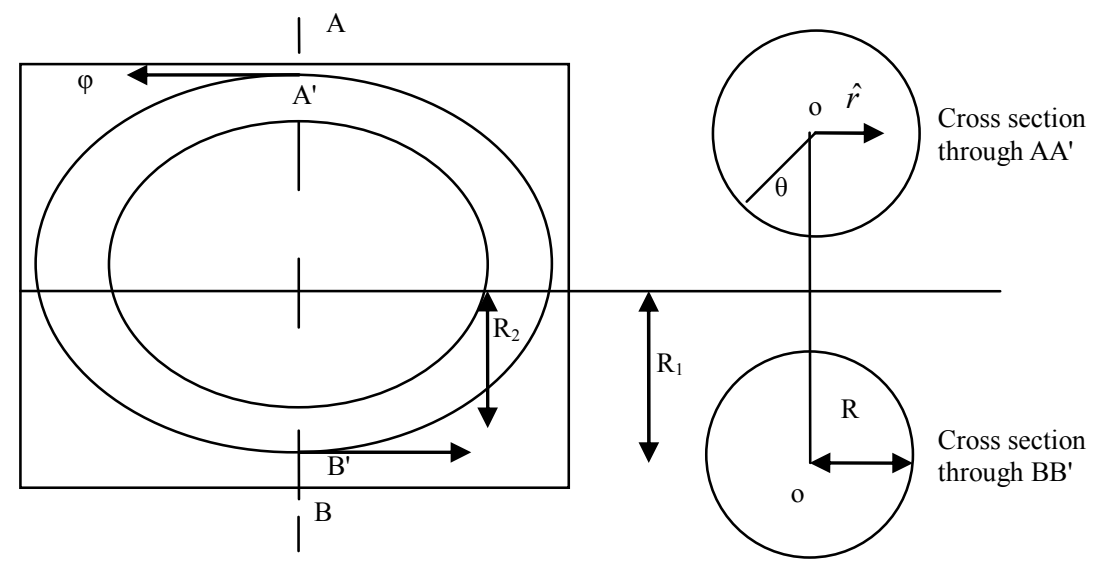

(a)

(b)

Figure 1. The physical model of the problem.

With theassumption of $H(r, \theta, \varphi)=R(r) Y(\theta, \varphi)$ and using separation variable method we have

$$
\begin{aligned}
& \frac{1}{R} \frac{\mathrm{d}}{\mathrm{d} r} r^{2} \frac{\mathrm{d} R}{\mathrm{~d} r}+k r^{2} \\
& =-\frac{1}{Y}\left[\frac{1}{\sin \theta} \frac{\partial}{\partial \theta} \sin \theta \frac{\partial}{\partial \theta}+\frac{1}{\sin ^{2} \theta} \frac{\partial^{2}}{\partial \phi^{2}}\right] Y(\theta, \phi)=\lambda
\end{aligned}
$$

where $\lambda$ is constant.

Therefore, the following equation is satisfied:

$$
\begin{gathered}
\frac{\mathrm{d}^{2} R}{\mathrm{~d} r^{2}}+\frac{2}{r} \frac{\mathrm{d} R}{\mathrm{~d} r}+\left(k-\frac{\lambda}{r^{2}}\right) R=0 \\
{\left[\frac{1}{\sin \theta} \frac{\mathrm{d}}{\mathrm{d} \theta} \sin \theta \frac{\mathrm{d}}{\mathrm{d} \theta}+\frac{1}{\sin ^{2} \theta} \frac{\mathrm{d}^{2}}{\mathrm{~d} \varphi^{2}}\right] Y(\theta, \varphi)=-\lambda Y(\theta, \varphi)}
\end{gathered}
$$

The solution of Equation (9) is expressed as follows:

$$
R(r)=C_{n} J_{n}(k r)+D_{n} Y_{n}(k r)
$$

Since the area of interest includes $r=0$ and $r \rightarrow 0$, $Y_{n}(k r) \rightarrow \infty, D_{n}=0$. From the Equation (11), we get

$$
R(r)=C_{n} J_{n}(k r)
$$

where $J_{n}(k r)$ is Bessel function.

The solution for (10) is simplified as follows:

$$
\begin{aligned}
& Y_{l}^{m}(\theta, \varphi) \\
& =\sqrt{(2 l+1) / 4 \pi} \sqrt{(l-m) ! /(l+m) !} P_{l}^{m}(\operatorname{Cos}(\theta)) \mathrm{e}^{i m \varphi}
\end{aligned}
$$

where $m=0 \rightarrow l, l=0,1,2,3,4, \cdots$ and $Y_{l}^{m}(\theta, \varphi)$ is a spherical harmonic function. $P_{l}^{m}(\cos \theta)$ is a Legendre's associated polynomial defined by the expression

$$
P_{l}^{m}(x)=\left(1-x^{2}\right)^{\frac{m}{2}} \frac{\mathrm{d}^{m}}{\mathrm{~d} x^{m}}\left[\frac{1}{2 ! l !} \frac{\mathrm{d}^{l}}{\mathrm{~d} x^{l}}\left(x^{2}-1\right)^{l}\right]
$$

where $x=\cos \theta$.
From Equations (12) and (13) we got the magnetic field as

$$
H(r, \theta, \varphi)=C_{n} J_{n}(k r) Y_{l}^{m}(\theta, \varphi),
$$

Based on the expression of $H(r, \theta, \varphi)$, the formula used to calculate the electrical field is deduced as follows: considering Equation (11) together with $J=\sigma E, D=\varepsilon E$, the following expression is deduced

$$
E(r, \theta, \varphi)=\frac{1}{\sigma+j \omega \varepsilon} \nabla \wedge H
$$

Assuming $E(r, \theta, \varphi)$ and $E_{\theta}(r, \theta, \varphi)$ represent the $\hat{\theta}$ and $\hat{r}$ components of the electrical field $E$ then

$$
\begin{aligned}
& E_{r}(r, \theta, \varphi)=\frac{1}{\sigma+j \omega \varepsilon} \frac{1}{r} \frac{\partial H}{\partial \theta} \\
& E_{\theta}(r, \theta, \varphi)=-\frac{1}{\sigma+j \omega \varepsilon} \frac{\partial H}{\partial r}
\end{aligned}
$$

From Equation (14) we find

$$
\frac{\partial H}{\partial r}=C_{n} J_{n}^{\prime}(k r) Y_{l}^{m}(\theta, \varphi),
$$

where

$$
J_{n}^{\prime}(k r)=\frac{1}{2} \sum_{s=0}^{\infty} \frac{(-1)^{s}}{s !} \frac{(n+2 s)}{\Gamma(n+s+1)}\left(\frac{k r}{2}\right)^{n+s-1}
$$

and

$$
\frac{\partial H}{\partial \theta}=C_{n} J_{n}(k r) \frac{\partial Y_{l}^{m}(\theta, \varphi)}{\partial \theta}
$$

From Equations (19)-(21) in (17) and (18) we have

$$
E_{r}(r, \theta, \varphi)=\frac{1}{\sigma+j \omega \varepsilon} \frac{1}{r} C_{n} J_{n}(k r) \frac{\partial Y_{l}^{m}(\theta, \varphi)}{\partial \theta}
$$

and

$$
E_{\theta}(r, \theta, \varphi)=\frac{1}{\sigma+j \omega \varepsilon} C_{n} J_{n}^{\prime}(k r) Y_{l}^{m}(\theta, \varphi)
$$




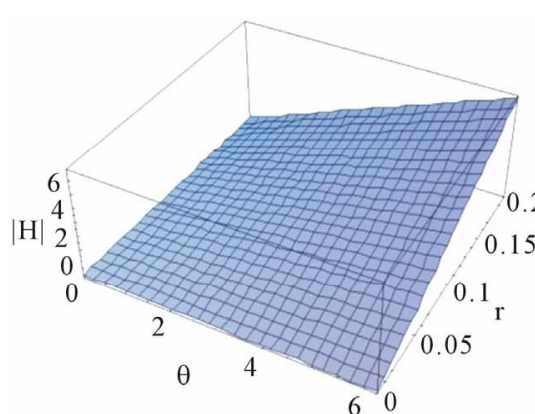

(a)

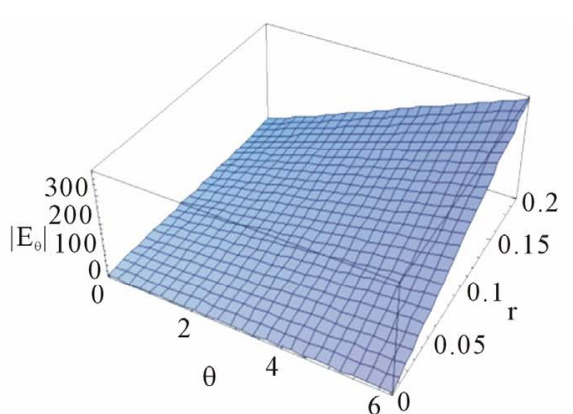

(b)

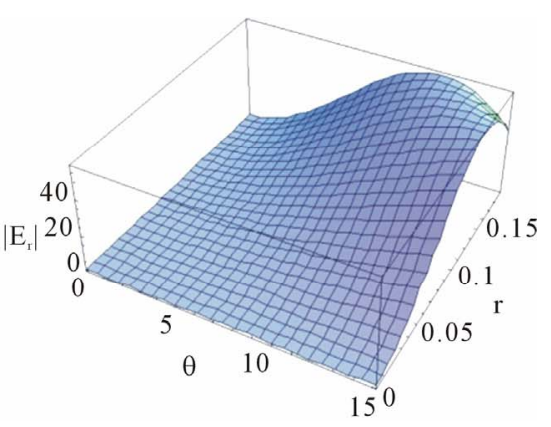

(c)

Figure 2. (a) Amplitude of $H$ at $f=5 \mathrm{kHz}$; (b) Amplitude of $E_{\theta}$ at $f=5 \mathrm{kHz}$; (c) Amplitude of $E_{r}$ at $f=5 \mathrm{kHz}$.

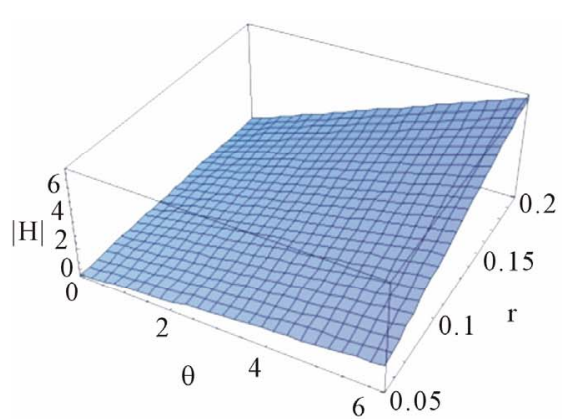

(a)

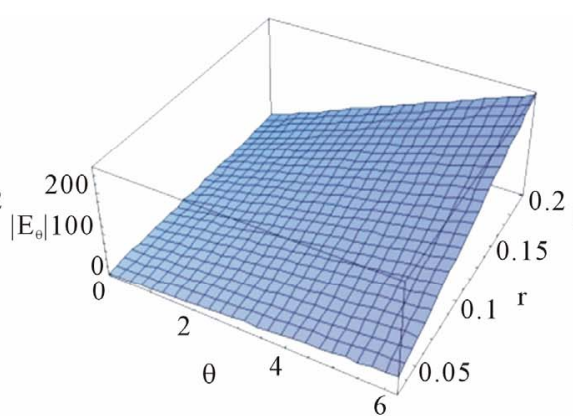

(b)

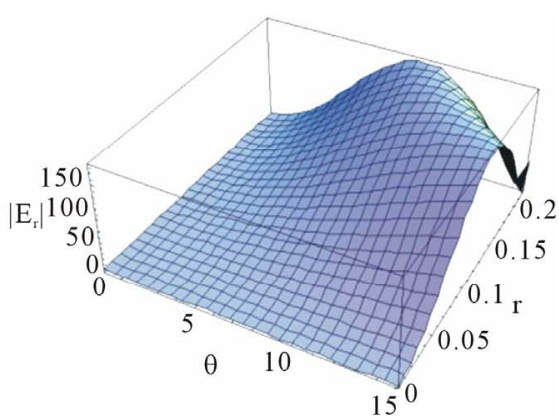

(c)

Figure 3. (a) Amplitude of $H$ at $f=300 \mathrm{kHz}$; (b) Amplitude of $E_{\theta}$ at $f=300 \mathrm{kHz}$; (c) Amplitude of $E_{r}$ at $f=300 \mathrm{kHz}$.

The electrical and magnetic fields in toroidal core have been calculated using Equations (15), (22) and (23) based upon the typical values $[9,10]$.

\section{Numerical Results}

The magnitude of the frequency rate of change of the electromagnetic field due to propagation in a spherical core is computed for different frequencies, and is shown in Figures 2(a)-(c) and 3(a)-(c). In these figures, the amplitude of the tangential component $\left(E_{\theta}\right)$ of electrical field is much larger than the normal component $\left(E_{r}\right)$ at high frequency. From the Figures 2(a) and 3(a), it is found that the magnetic field strength in the core is very small when $r$ is small. With the increase of $r$, the magnetic intensity increases too. The magnetic field strength reaches the maximal value when $r=R$ as shown in Figure 3(a), $\theta=0$. From Figures 3(b) and (c), we can reach the conclusion that the components of the electrical field intensity increase with the increase of radius at same angle $\theta$.

The $E_{\theta}$ component, it reaches the maximal value at $r$ $=R$ and $\theta=2 \pi$.

However for the normal component, it reaches the maximal value somewhere $\pi<\theta<2 \pi$. With the increase of frequency, this phenomenon becomes clearer. Based on the equations of electrical and magnetic fields, core losses are calculated in this paper.

\section{Conclusion}

The distributions of the electrical and magnetic fields in the toroidal core with spherical cross section have been calculated based on Maxwell's equations. Based on the equations of electrical and magnetic fields core losses were calculated. It is found that the losses obtained by taking the boundary conditions into consideration are larger than those without considering it.

\section{REFERENCES}

[1] H. Saotome and Y. Sakaki, "Iron Loss Analysis of $\mathrm{M}_{\mathrm{n}}-\mathrm{Z}_{\mathrm{n}}$ Ferrite Cores," IEEE Transactions on Magnetics, Vol. 33, No. 1, 1997, pp. 728-734. doi:10.1109/20.560105

[2] C. F. Foo, D. M. Zhang and X. Li, "A Simple Approach for Determining Core-Loss of Magnetic Materials," Journal of the Magnetics Society of Japan, Vol. 22, 1998, pp. 277-279.

[3] O. M. Abo-Seida and S. T. Bishay, "Response above a Plane-Conducting Earth to a Pulsed Vertical Magnetic Dipole at the Surface," Canadian Journal of Physics, Vol. 78, No. 9, 2000, pp. 883-844. doi:10.1139/p00-050

[4] O. M. Abo-Seida and G. M. Sami, "Transient Fields of a Vertical Electric Dipole on an M-Layered Dielectric Medium," Canadian Journal of Physics, Vol. 81, No. 6, 2003, p. 869.

[5] S. T. Bishay, O. M. Abo-Seida and G. M. Sami, "Transient Electromagnetic Field of a Vertical Magnetic Dipole 
on a Two-Layer Conducting Earth," IEEE Transactions on Geoscience and Remote Sensing, Vol. 39, No. 4, 2001, pp. 894-897. doi:10.1109/36.917919

[6] S. T. Bishay and G. M. Sami, "Time-Domain Study of the Transient Fields for a Thin Circular Loop Antenna," $\mathrm{Ca}$ nadian Journal of Physics, Vol. 80, No. 9, 2002, pp. 9551003. doi:10.1139/p02-049

[7] O. M. Abo-Seida, "Far-Field Due to a Vertical Magnetic Dipole in Sea," Applied Electromagnetic Waves and Application, Vol. 20, No. 6, 2006, pp. 707-715.

[8] J. R. Wait, "EM Fields of a Phased Line Current over a
Conducting Half-Space," IEEE Electromagnetic Compatibility Society, Vol. 38, No. 4, 1996, pp. 608-611. doi:10.1109/15.544318

[9] G. R. Skutt and F. C. Lee, "Characterization of Dimensional Effects in Ferrite-Core Magnetic Devices," IEEE Transactions on Magnetics, Vol. 2, 1996, pp. 1435-1440.

[10] Y. Sakaki, M. Yoshida and T. Sato, "Formula for Dynamic Power Loss in Ferrite Cores Taking into Account Displacement Current," IEEE Transactions on Magnetics, Vol. 29, 1993, pp. 3517-3519. doi:10.1109/20.281215 